\title{
Emerging Roles of Dysregulated MicroRNAs in Myasthenia Gravis
}

\author{
Lin Wang ${ }^{1}$ and Lijuan Zhang ${ }^{2 *}$ \\ ${ }^{1}$ Department of Emergency Medicine, Shengjing Hospital of China Medical University, Shenyang, China, ${ }^{2}$ Department of \\ Obstetrics and Gynecology, Shengjing Hospital of China Medical University, Shenyang, China
}

Myasthenia gravis (MG) is a rare acquired autoimmune neuromuscular disease. Autoantibodies, cellular immunity, complement, and cytokines are involved in the pathogenesis of MG. It is characterized by the dysfunction of neuromuscular junction transmission and skeletal muscle weakness. MicroRNAs (miRNAs) are non-coding small molecule ribonucleic acids that regulate various biological processes (e.g., development, differentiation, and immunity) at the transcriptional and post-transcriptional levels of gene expression. miRNAs play an important regulatory role in the pathogenesis of autoimmune diseases, including MG. In recent studies, the functional mechanisms underlying the role of miRNAs in the pathogenesis of $M G$ have received increasing

OPEN ACCESS

Edited by: Arumugam R. Jayakumar, Miami VA Healthcare System, United States

Reviewed by:

Ravindran Caspa Gokulan, University of Miami Health System, United States

Kannappan Sriramajayam, University of Miami Health System, United States

*Correspondence: Lijuan Zhang

cmu_lijuanzhang@163.com

Specialty section:

This article was submitted to

Neurodegeneration,

a section of the journal

Frontiers in Neuroscience

Received: 05 December 2019

Accepted: 22 April 2020

Published: 21 May 2020

Citation:

Wang L and Zhang L (2020) Emerging Roles of Dysregulated MicroRNAs in Myasthenia Gravis.

Front. Neurosci. 14:507. doi: 10.3389/fnins.2020.00507 attention. miRNAs are highly stable and have high specificity in peripheral body fluids. Therefore, the miRNAs in body fluids may represent promising biomarkers for determining the prognosis of $M G$ and the efficacy of treatment. This article reviews the role of miRNAs in the pathogenesis of MG, highlights the potential of miRNAs as new biomarkers for the diagnosis of $M G$, and deepens our understanding of disease processes.

Keywords: microRNA, myasthenia gravis, autoimmune, neuromuscular disease, interleukin

\section{INTRODUCTION}

Myasthenia gravis (MG) is an autoimmune disease that is primarily mediated by antibodies against the acetylcholine receptor (AChR) on the postsynaptic membrane of the neuromuscular junction (NMJ) and muscle-specific tyrosine kinase (MuSK), which leads to postsynaptic membrane transmission dysfunction involving both cellular immunity and complement (Gilhus and Verschuuren, 2015; Gilhus, 2016). The annual incidence of MG is about 0.25 to 2 per 100,000 people, mostly in adults (Vincent et al., 2001; Harris et al., 2019). The onset of MG is insidious, and the course of the disease is prolonged. The clinical characteristics of this disease are partial or complete skeletal muscle weakness that can be aggravated by activity. Symptoms are relieved by rest or treatment with cholinesterase inhibitors (ChEI) (Pohanka, 2019; Souto et al., 2019). In severe cases, the medullary and respiratory muscles may be involved, leading to myasthenia crisis (Roper et al., 2017; Barnett et al., 2019).

An abnormal immune response in the thymus is closely associated with the occurrence and development of MG. About $80 \%$ of MG patients have thymic hyperplasia or tumors, and a variety of cellular components that induce and maintain abnormal AChR immune responses are located in the proliferative thymus tissue of MG patients (Niendorf et al., 2005; Cordiglieri et al., 2014). The pathogenic mechanism mediated by AChR antibodies (AChR-Ab) includes: (1) AChR-Ab binding 
to the AChR, activation of complement, and $\mathrm{AChR}$ destruction; (2) blocking of the acetylcholine binding site in the AChR by AChR-Ab; (3) crosslinking of AChR by AChR-Ab to accelerate the rate of AChR degradation (Kohler et al., 2017; Huang et al., 2018). Other related pathogenic antibodies identified in MG include MuSK antibodies (MuSK-Ab), low-density lipoprotein receptor (LDLR)-related protein 4 antibodies (LRP4-Ab), and ryanodine receptor antibodies (RyR-Ab) (Morren and Li, 2018). MuSK is a transmembrane protein located on the postsynaptic membrane that can coexist with AChR. A complex between agrin and MuSK can assemble AChR in the postsynaptic membrane. MuSK-Abs inhibit the agrin-MuSK signal, which decreases the concentration of $\mathrm{AChR}$ and alters the stability of the NMJ (Huijbers et al., 2019). LRP4, located in the postsynaptic membrane, activates MuSK by binding to the LRP4 receptor to initiate a signaling cascade that promotes the aggregation of AChR. RyR-Abs do not directly cause muscle weakness but may serve as biomarkers of MG (Mantegazza and Antozzi, 2018; Ohnari et al., 2018; Park et al., 2018).

MicroRNAs (miRNAs) are conserved single-stranded noncoding RNAs that bind target mRNAs to regulate gene expression at the post-transcriptional level through the inhibition of translation or degradation of mRNA (Michlewski and Caceres, 2019). More than $30 \%$ of the genes in the human genome are thought to be regulated by miRNAs. These genes function in cell proliferation, maturation, differentiation, apoptosis, and other life processes (e.g., immune regulation) (Carthew and Sontheimer, 2009). In recent years, studies have demonstrated that the pathogenesis of $\mathrm{MG}$ is associated with thymus abnormalities and multiple genetic and environmental factors; abnormal miRNA function may also be involved in the pathological process of MG (Bo et al., 2019). For example, miR146 expression was significantly upregulated in MG patients and accompanied by high TLR4, CD40, and CD80 expression levels in AChR-specific B cells (Lu et al., 2013), miR-15a appeared to be involved in the regulation of inflammatory cytokine expression in MG patients (Liu et al., 2016) and miR-125a-5p in the thymus may negatively regulate Foxp expression, leading to an imbalance in autoimmune regulation and MG pathogenesis (Li et al., 2016).

Circulating miRNAs are highly stable and specific and difficult to degrade. Detection of circulating miRNAs can guide the diagnosis and treatment of diseases and provide information on prognosis (Hajjari et al., 2017; Jin and Xing, 2017). Studies have demonstrated that miR-150-5p and miR-21-5p levels are increased in AChR-Ab + MG patients (Punga et al., 2015), suggesting that these two miRNAs may represent promising serum biomarkers for this subset of MG patients. miRNAs are involved in multiple complex and delicate regulatory mechanisms, making the entire signaling pathway a promising therapeutic agent (miRNA mimics) or target (anti-miRNA) in a variety of pathological processes (Kim et al., 2018). At the same time, the advancement of RNA molecular delivery technology has made miRNA-based treatment programs more feasible (Zhao et al., 2015; Wang et al., 2016).

As a relatively rare chronic autoimmune disease of the nervous system, the pathogenesis of MG has not been fully elucidated. In recent years, a number of studies have revealed that dysregulated
miRNAs may mediate the pathogenesis of MG through a variety of pathways. In this review, we discuss the emerging roles of dysregulated miRNAs in the pathogenesis of MG in order to provide new ideas for the early diagnosis and introduce miRNAbased therapeutic approaches.

\section{BIOLOGICAL ORIGIN AND REGULATORY MECHANISM OF MIRNAs}

miRNAs are small single-stranded RNAs of approximately 22 nucleotides (NTs). Although they do not encode proteins, miRNAs can regulate target gene expression and cell function after transcription via an epigenetic mechanism (Ha and Kim, 2014). These molecules can not only bind the $3^{\prime}$ untranslated region ( $3^{\prime}$-UTR) but also bind the $5^{\prime}$-UTR of mRNA for post-transcriptional regulation of target genes. Through these interactions, miRNAs can regulate cell proliferation, differentiation, and apoptosis, and perform other regulatory roles at various stages of growth and development of organisms (Ferrante and Conti, 2017).

miRNA is thought to be associated with the mechanisms of immunoregulation and activation, including the T-cell response, inflammatory reaction, and expression of inflammatory factors. The study of miRNAs specific to MG may provide valuable information for the pathogenesis and treatment of this disease (Josefowicz et al., 2012; Li et al., 2016; Colamatteo et al., 2019). Dysregulation of miRNA expression and its role in disease can occur as follows: (1) downregulation or deletion of miRNA expression - gene mutation or downregulated or abnormal transcription; (2) miRNA overexpression - overexpression due to gene amplification or mutation inhibits the production of target proteins; (3) Mutation in the $3^{\prime}$-UTR of mRNA affects the miRNA binding site; (4) Mutation in the UTR of mRNA forms a new miRNA binding site, which inhibits the function of affected genes (Quinlan et al., 2017; Fu et al., 2019; Patel et al., 2019).

\section{THE ROLE OF miRNAS IN MG PATHOGENESIS}

Several studies using next-generation gene sequencing and high-throughput techniques identified differential expression of miRNAs in the thymus of MG patients. Thymic hyperplasia with ectopic germinal center (GC) is a characteristic pathological change found in early MG (Zuckerman et al., 2010). Sengupta et al. (2018) identified 38 differentially expressed miRNAs in GC-positive patients, two downregulated miRNAs (miR-139$5 p$ and miR-452-5p) were identified by quantitative real-time PCR, and validated a negative regulatory relationship with Regulator of G protein Signaling 13 (RGS13), suggesting their involvement in support of GC formation in the thymus. Cron et al. (2018) identified 61 differentially expressed miRNAs (24 up- and 37 downregulated) in a study involving $20 \mathrm{MG}$ patients and five controls. Among them, miR-7-5p and miR-125a-5p were confirmed by qRT-PCR. miR-7 was significantly downregulated and inversely correlated with CCL21 mRNA expression, while 
TABLE 1 | Summary of altered microRNAs (miRNAs) and their targeted cytokines in Myasthenia Gravis (MG).

\begin{tabular}{|c|c|c|c|c|c|}
\hline MiRNAs & Immune cells type & Regulation & Target interleukin & Roles & References \\
\hline miR-181a & Th1 cell & Down-regulated & $\mathrm{IL}-2 \uparrow$ & $\begin{array}{l}\text { Modulate the activation of CD } 4+T \text { cells, } \\
\text { expression of transcription factors related to } \\
\text { Th1 and Th17 cells. }\end{array}$ & Liu et al. (2019) \\
\hline miR-20b & Th1 cell & Down-regulated & $\mathrm{IL}-8 \uparrow, \mid \mathrm{IL}-25 \uparrow$ & Inhibit the expression of inflammatory cytokines. & Chunjie et al. (2015) \\
\hline miR-320a & Th1 cell & Down-regulated & $\mathrm{IL}-2 \uparrow$ & $\begin{array}{l}\text { miR-320a can regulate COX-2 expression } \\
\text { through ERK/NF-кB pathways }\end{array}$ & Cheng et al. (2013) \\
\hline miR-15b & Th1 cell & Down-regulated & IL-15个 & $\begin{array}{l}\text { Regulates IL-15 expression by directly targeting } \\
\text { its } 3^{\prime} \text {-UTR }\end{array}$ & Shi et al. (2015) \\
\hline let-7c & Th2 cell & Down-regulated & $\operatorname{IL}-10 \uparrow$ & $\begin{array}{l}\text { Regulates IL-10 expression by directly targeting } \\
\text { its } 3^{\prime} \text {-UTR }\end{array}$ & Jiang et al. (2012) \\
\hline $\mathrm{miR}-181 \mathrm{c}$ & Th17 cell & Down-regulated & $\mathrm{IL}-7 \uparrow, \mathrm{IL}-17 \uparrow$ & Negatively regulate immune cell activation & Zhang et al. (2016) \\
\hline miR-15a & Th17 cell & Down-regulated & $\mathrm{IL}-17 \uparrow, \mathrm{IFN}-\gamma \uparrow$ & $\begin{array}{l}\text { Modulate CXCL10 to increase the expression of } \\
\text { cytokines }\end{array}$ & Liu et al. (2016) \\
\hline miR-145 & B cells & Up-regulated & CD28 $\downarrow$ & $\begin{array}{l}\text { Play an important role in antigen specific T cells } \\
\text { activation }\end{array}$ & Wang et al. (2013) \\
\hline $\mathrm{miR}-146$ & B cells & Down-regulated & $\mathrm{CD} 40 \uparrow, \mathrm{CD} 80 \uparrow$ & $\begin{array}{l}\text { Modulate differentiation and function of cells in } \\
\text { innate as well as adaptive immunity }\end{array}$ & Lu et al. (2013) \\
\hline
\end{tabular}

miR-125a was upregulated and associated with FOXPS expression (Cron et al., 2018).

CXCL13 is a target for the miRNA miR-548k (Li et al., 2018). Dysregulation of miR-548k has been observed in the thymus hyperplasia of MG patients. Thus, dysregulated miR548k may contribute to MG pathogenesis by regulating CXCL13 mRNA levels (Li et al., 2018). miR-653 was reported decreased in thymocyte and had a negative regulation with tripartite motif 9 (TRIM9), Cao et al. (2019) suggested miR-653 impair proliferation and promote apoptosis of thymocytes of MG mice by suppressing TRIM9. Current studies on the potential role of dysregulated miRNAs in MG pathogenesis are focused on their effect on different MG antibodies and various cytokines secreted by immune cells in this disease. In the next sections, we will discuss the different pathological roles of miRNAs in MG and highlight the potential clinical use of miRNAs as biomarkers or therapeutic targets (Table $\mathbf{1}$ and Figure 1).

\section{THE ROLE OF miRNAs IN MG-RELATED ANTIBODIES}

The AChR is present on the surface of muscle cells and concentrated in the synapses between nerve and muscle cells (Bruhova and Auerbach, 2017). Antibodies against the AChR of the postsynaptic NMJ were detected in approximately $80 \%$ of MG patients (Cavalcante et al., 2012; Tuzun et al., 2012). Nogales-Gadea et al. (2014) evaluated the levels of miRNAs in the serum of three subgroups of 15 AChR-Ab-positive MG patients (early-onset MG, late-onset MG, and thymoma). They identified 32 differentially expressed miRNAs. miR-15b was expressed at low levels in all three groups, whereas miR-122, miR-140-3p, miR-185, miR-192, and miR-20b were expressed at different levels in early- and late-onset MG compared to the controls (Nogales-Gadea et al., 2014). In a separate study of 19 AChR-positive early-onset MG (AChR-EOMG) patients and 12 controls, Barzago et al. (2016) demonstrated that miR-612, miR3651, and miR-3653 were upregulated in the peripheral blood mononuclear cells (PBMCs) of these AChR-EOMG patients, suggesting that these dysregulated miRNAs may be involved in the pathogenesis of AChR-EOMG (Barzago et al., 2016). miR146 is another dysregulated miRNA found in the PBMCs from MG patients with AChR-Ab-positive disease (REF). miR-146 expression was significantly upregulated in MG patients and accompanied by high TLR4, CD40, and CD80 expression levels in AChR-specific B cells (Lu et al., 2013). Based on these results, the authors suggest that the dysregulation of miR-146 could be involved in MG pathogenesis through the regulation of AChRAb-specific B cells (Lu et al., 2013). miR-145 was downregulated in the PBMCs from MG patients and CD4+ CD25- T cells from experimental autoimmune MG (EAMG) rats (Wang et al., 2013).

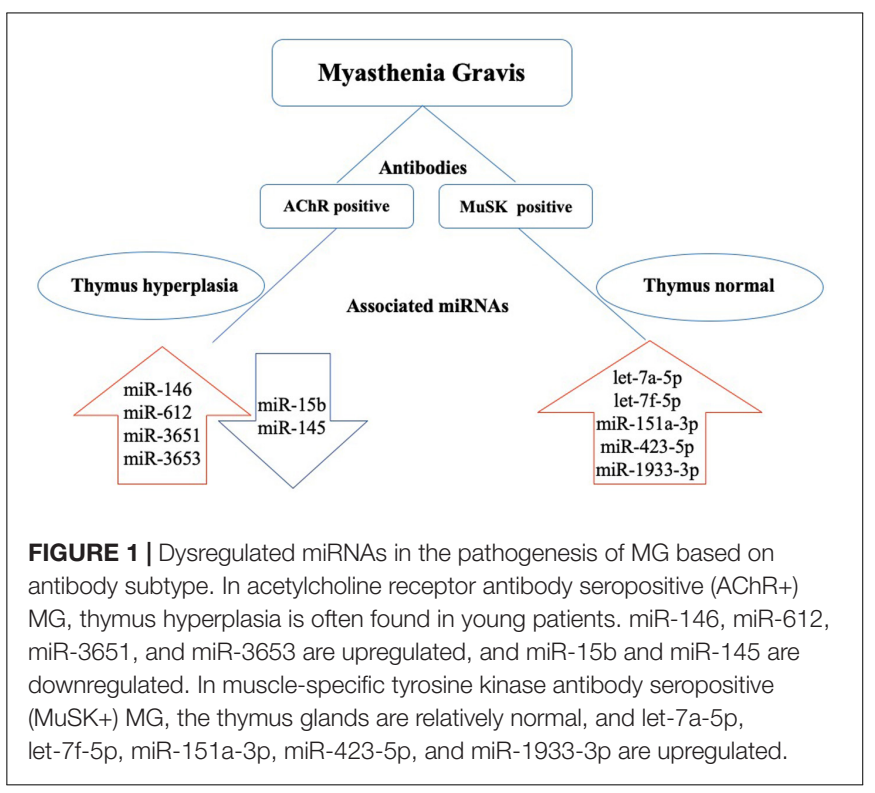


CD28 is a target of miR-145 and its levels can be reduced by the upregulation of miR-145 expression. In addition, overexpression of miR-145 can suppress the expression of NFATc1 in AChRAb-specific CD4+ T cells and decrease the severity of MG by reducing IL-17 production (Wang et al., 2013).

MuSK is involved in the induction of AChR aggregation during the development of the NMJ, which is crucial for the formation of neuromuscular synapses. MuSK plays an important role in maintaining the homeostasis of the postsynaptic membrane after the NMJ matures. It is speculated that MuSK$\mathrm{Ab}$ cause a decrease in the AChR levels in the postsynaptic membrane by altering MuSK function (Hoch et al., 2001). Previous studies showed that MuSK-Abs are found in 1 to $10 \%$ of MG patients and more common in European and American female MG patients. MuSK-Ab-positive MG patients are prone to facial, bulbar, and respiratory muscle weakness that is often accompanied by muscle atrophy. MuSK-Ab-positive MG usually presents with more severe clinical symptoms and is less sensitive to treatment compared to AChR antibodypositive MG (Guptill et al., 2011; Chang et al., 2014; Gilhus and Verschuuren, 2015). Although the incidence of MuSK-Abpositive MG is relatively low, there have still been a few studies investigating miRNA dysregulation in this type of MG. Bogatikov et al. (2019) identified 13 dysregulated miRNAs (11 upregulated and 2 downregulated) in the omohyoid muscle of mice with MuSK-Ab-positive EAMG. Among these differentially expressed miRNAs, miR-1930-5p and miR-1933-3p had a greater effect on the expression of target genes than other miRNAs, including Impal (inositol monophosphatase I) and Mrpl27 (mitochondrial ribosomal protein L27). In addition to these two miRNAs, let$7 a-5 p$, let-7f-5p, mir-151a-3p, and mir-423-5p are specifically elevated in MuSK-Ab-positive MG patients (Punga et al., 2016).

LRP4 is a member of the LDLR family and is the receptor for proteins that are critical for MuSK activation, AChR aggregation, and NMJ formation (Barik et al., 2014). LRP4 antibodies (LRP4Abs) are detected in 2 to $45 \%$ of patients with double seronegative MG (Sieb, 2014). Shen et al. (2013) first reported LRP4-Abs in the blood of patients with MG. Injection of LRP4-Abs into healthy mice caused typical MG symptoms, validating the involvement of LRP4-Abs in the pathogenesis of MG (Shen et al., 2013). Few studies have focused on the relationship between dysregulated miRNAs and LRP4-Abs. LRP4 is a target for miR-490-3p (Xie et al., 2019). It can also be a target of miR-196a, miR-1232, and miR-1544; however, there are no studies on the relationship between these miRNAs and LRP4 in MG (Li et al., 2015; Karakatsani et al., 2017).

Agrin is a basement membrane protein that includes two isoforms, neuronal agrin ( $\mathrm{N}$-agrin) and muscular agrin (M-agrin). N-agrin binds to LRP4-Ab, activates MuSK$\mathrm{Ab}$, induces the aggregation of AChR-Abs, and plays an important role in NMJ structure formation and maintenance (Koneczny and Herbst, 2019). Titin antibodies are raised against the major immunogenic region (MIR) at the A/I band junction. The effects of the Titin antibodies are closely related to $\mathrm{MG}$ that is complicated with thymoma, and these antibodies are important for the diagnosis of this subtype of MG (Szczudlik et al., 2014). At present, the relationship between miRNAs and these two antibodies remains to be studied.

\section{miRNAs AND MG-RELATED IMMUNE CELLS AND CYTOKINES}

CD4+ T cells, also known as helper T cells (Th), usually include three subpopulations of cells (Th1, Th2, and Th3). In MG patients, Th1 and Th2 cells can act on AChR-Ab-specific B cells by secreting cytokines to enhance the humoral immune response, while Th3 cells mainly exert inhibitory effects by secreting transforming growth factor- $\beta$ (TGF- $\beta$ ) (Pianta et al., 2015). Mature B cells can be stimulated by antigen by peripheral lymphatic cells. With the help of Th and antigen-presenting cells and the cytokines produced by them, B cells can be activated to proliferate and differentiate into plasma cells that synthesize and secrete antibodies. For example, Cron et al. (2019) demonstrated that miR-150 expression was downregulated in the PBMCs from MG patients, especially in CD4+ T cells. They also observed upregulated miR-150 expression in the thymus, which was associated with the involvement of thymic B cells in MG (Cron et al., 2019).

Th1 cell-specific cytokines include interleukin-2 (IL-2), IL12 , interferon- $\gamma$ (IFN- $\gamma$ ), and tumor necrosis factor- $\alpha$ (TNF$\alpha$ ), which initiate cellular immune responses (Buonocore et al., 2019). IL-2 is a cytokine that plays an immune-enhancing role by inducing the proliferation of Th1 cells and producing IFN- $\gamma$ to promote B-cell proliferation and the secretion of antibodies (Buonocore et al., 2019). miR-181a is downregulated in PBMCs from MG patients and related to the levels of AChR antibody. miR-181a is involved in the modulation of CD4+ T-cell activation and associated with the expression of transcription factors related to Th1 and Th17. Liu et al. (2019) found that IL-2 is regulated by miR-181a. There is a negative correlation between the levels of miR-181a and IL-2, suggesting that miR-181a is involved in the pathogenesis of MG through the modulation of IL-2 expression (Liu et al., 2019). IL-8 is a chemotactic cytokine that promotes inflammatory cell chemotaxis and induces cell proliferation. IL- 8 is secreted by Th1 cells, IL- 8 is secreted by Th1 cells and mediate cytotoxic and local inflammationrelated immune response-assisted antibody production. Its main biological functions are to activate neutrophils and promote neutrophil lysosomal enzyme activity and phagocytosis (Meniailo et al., 2018). IL-25 is a new member of the IL-17 family. It plays an important regulatory role in type 2 immune reaction. Chunjie et al. (2015) found that the levels of IL-8 and IL-25 are increased in the serum from MG patients. They also found that the expression of miR-20b was downregulated in these MG patients. There was a direct negative relationship between miR$20 \mathrm{~b}$ and IL- 8 and IL-25. The down-regulated miR-20b expression could altered the serum IL-8 and IL-25 levels, exacerbating cytotoxic and local inflammation-related immune responseassisted antibody production (Chunjie et al., 2015). Cheng et al. (2013) found that miR-320a is downregulated in MG patients. Their data suggest that miR-320a is involved in regulating the extracellular-regulated protein kinase (ERK)/nuclear factor 
kappa-B (NF- $\kappa \mathrm{B})$ pathway, which in turn induces the expression of cyclooxygenase 2 (COX-2) and promotes the expression of IL2 and IFN- $\gamma$ (Cheng et al., 2013). Together, these effects may contribute to MG pathogenesis. IL-15 is produced by a variety of cells (e.g., activated monocytes and macrophages, epidermal cells, and fibroblasts). IL-15 induces B-cell proliferation and differentiation, stimulates T-cell and NK-cell proliferation, and also stimulates NK cells to produce IFN- $\gamma$ (Hromadnikova et al., 2016). The expression of IL-15 is significantly higher in patients with eye MG, limb MG, or thymomas compared to healthy controls. miR-15b is downregulated in MG patients. Shi et al. (2015) suggested that downregulated miR-15b targets IL-15 and negatively regulates its expression, to induce B-, $\mathrm{T}$-, and NK-cell proliferation and differentiation, and involves in $\mathrm{MG}$ progression.

Th2 cells mainly secrete IL-4, IL-6, IL-9, and IL-10, which promote B-cell proliferation, differentiation, and antibody formation and initiate humoral immunity. IL-10 levels are significantly higher in MG patients compared to controls and correlate with the clinical severity of MG (Yeh et al., 2009). Jiang et al. (2012) found that the let-7 family of miRNAs was downregulated in the PBMCs from MG patients. IL-10 is a target of let-7c, and its expression levels are inversely correlated with that of let-7c (Jiang et al., 2012). Thus, dysregulated let-7c expression may contribute to the initiation and progression of MG through its regulation of IL-10 (Jiang et al., 2012).

The relative lack of Treg cells is a common feature of human autoimmune diseases. The thymus produces most of the Treg cells in the human body. Forkhead box P3 (Foxp3) is a key transcription regulator of the immunosuppressive function of Treg cells. Downregulation of Foxp3 expression can lead to the loss of Treg cell function (Kumar et al., 2019). Li et al. (2016) found that miR-125a-5p is highly expressed in the thymus of MG patients with thymomas, and Foxp is a target gene for miR-125a5p. In MG patients with thymoma, miR-125a-5p in the thymus may negatively regulate Foxp expression, leading to an imbalance in autoimmune regulation and MG pathogenesis (Li et al., 2016).

In addition to CD4+ $\mathrm{T}$ cells, Th17 cells represent another cell subpopulation involved in the pathogenesis of MG. IL-7 is a multipotent cytokine that is required for the growth of $\mathrm{T}$ and $B$ cells and resistance to apoptosis. It can maintain the stability of the immune system and promote the proliferation and differentiation of $\mathrm{CD} 4+$ and $\mathrm{CD} 8+\mathrm{T}$ cell subsets in the thymus (Vanhanen et al., 2018). As an important cytokine secreted by Th17 cells, IL-17 can induce the expression of various chemokines and cytokines, participate in immune cell and inflammatory responses, and play an important role in the pathogenesis of autoimmune diseases (Khan and Ghazanfar, 2018). Increased IL-7 and IL-17 serum levels have been found in MG patients (REF). Zhang et al. (2016) demonstrated that miR-181c is downregulated in the PBMCs from MG patients and is negatively correlated with the levels of both IL-7 and IL-17. Increased expression of miR-181c caused a decrease in the IL-7 and IL-17 levels released from cultured PBMCs, relieving immune cell-mediated inflammation (Zhang et al., 2016). miR-15a appears to be involved in the regulation of inflammatory cytokine expression in MG patients (Liu et al., 2016). Interferon-gamma-inducible protein 10 (CXCL10) is a direct target gene for miR-15a in MG. The downregulation of miR-15a expression leads to the upregulation of CXCL10 expression and subsequent increases in the expression of IL-17 and IFN- $\gamma$ (Liu et al., 2016).

$\mathrm{B}$ cells originate from the bone marrow and enter all peripheral lymphoid organs of the human body as part of the humoral immune system. Dysregulation of miRNAs can lead to changes in the number of B cells, functional defects, or overactivation. It also causes the disorder of complement and cell surface molecules, resulting in the release of a large number of autoantibodies that attack the NMJ and participate in the pathogenesis of MG. CD28 is a target for miR-145 and can be reduced by the upregulation of miR-145 expression in EAMG rats (Wang et al., 2013). CD40 and CD80 are highly expressed in AChR-specific $\mathrm{B}$ cells and are regulated by dysregulated miR-146 expression, suggesting miR-146 may participate importantly in the regulation of AchR specific B cells and involve in the pathogenesis of MG (Lu et al., 2013).

\section{miRNAs AS BIOMARKERS OF MG}

Serum AChR, Titin, RyR, and MuSK antibodies have all been implicated in the pathogenesis of MG. Changes in their levels are helpful for the diagnosis of MG. However, the positive rate for the AChR antibodies in MG patients without thymoma is 36.8\% (Meriggioli and Sanders, 2012). The positive rate of serum Titin antibodies in MG patients is 27\% (Szczudlik et al., 2014). Although this positive rate may be helpful for early diagnosis, changes in Titin antibody levels fail to reflect the progression of the disease or its response to treatment (Szczudlik et al., 2014).

Circulating miRNAs are stable molecules in the blood that can be detected by non-invasive, highly sensitive methods. Previous studies demonstrated that many miRNAs could be used as biomarkers for disease diagnosis, including in MG. Several studies demonstrated that miR-150-5p and miR-21-5p levels are increased in AChR-Ab + MG patients (Punga et al., 2015), suggesting that these two miRNAs may represent promising serum biomarkers for this subset of MG patients. The levels of miR-150-5p may also correlate with MG severity. In contrast, miR-20b is downregulated in AChR+ MG patients compared to healthy controls (Chunjie et al., 2015). The levels of the let7 family of miRNAs are elevated in MuSK-Ab + MG patients (Punga et al., 2016; Punga and Punga, 2018). Plasma miR-210$3 \mathrm{p}$ and miR-324-3p levels are decreased in MuSK+ MG patients compared to healthy controls (Sabre et al., 2018a).

In addition to their diagnostic value, miRNAs may also be predictive of the prognosis of MG patients. It was reported that the miR-30e-5p levels are elevated in ocular MG patients and had high sensitivity in differentiating ocular MG and secondary generalized MG of late-onset ocular MG patients (Sabre et al., 2019). Sabre et al. (2018b) found that the levels of miR-21-5p and miR-150-5p were higher in generalized lateonset MG (LOMG) compared to ocular LOMG and positively correlated with the age of the MG patients. miR-21-5p, miR$30 \mathrm{e}-5 \mathrm{p}$, and miR-150-5p levels were decreased concurrently 
with clinical improvement after immunosuppression therapy, and their levels were positively correlated with the clinical MG composite score (Sabre et al., 2018b). miR-150-5p decreased 24 months after thymectomy, providing new evidence and further support for miR-150-5p as a biomarker for MG (Molin et al., 2018). Cavalcante et al. (2019) identified miR-323b-3p, miR-409-3p, and miR-485-3p as predictive biomarkers for the responsiveness of MG patients to immunosuppressive drugs. miR-181d-5p, miR-323b-3p, miR-340-3p miR-409-3p, and miR485-3p have also been validated as drug efficacy biomarkers (Cavalcante et al., 2019).

\section{mIRNA-BASED THERAPEUTIC APPROACHES FOR MG}

Biological drugs, such as immunosuppressants, have broad clinical prospects in the treatment of MG. Immunosuppressants currently used include monoclonal antibodies (e.g., eculizumab, rituximab, and belimumab) and the immunoglobulin regulator Efgartigimod (Mantegazza et al., 2018).

miRNAs are involved in MG immune disorders through a variety of mechanisms. Modulating the expression of pathogenic genes or inhibiting the activation of immune cells and the release of inflammatory cytokines may represent a novel strategy to treat MG. For example, overexpression of miR-146a in exosomes from dendritic cells was recently found to have antigen-specific suppressive effects in EAMG (Yin et al., 2017). miR-155 is a better-characterized miRNA that is involved in inflammation and the immune reactions observed in MG. Wang et al. (2014)

\section{REFERENCES}

Barik, A., Lu, Y., Sathyamurthy, A., Bowman, A., Shen, C., Li, L., et al. (2014). LRP4 is critical for neuromuscular junction maintenance. J. Neurosci. 34, 13892-13905. doi: 10.1523/JNEUROSCI.1733-14.2014

Barnett, C., Tabasinejad, R., and Bril, V. (2019). Current pharmacotherapeutic options for myasthenia gravis. Expert Opin. Pharmacother. 20, 2295-2303. doi: 10.1080/14656566.2019.1682548

Barzago, C., Lum, J., Cavalcante, P., Srinivasan, K. G., Faggiani, E., Camera, G., et al. (2016). A novel infection- and inflammation-associated molecular signature in peripheral blood of myasthenia gravis patients. Immunobiology 221, 1227-1236. doi: 10.1016/j.imbio.2016.06.012

Bo, C., Wang, J., Zhang, H., Cao, Y., Lu, X., Wang, T., et al. (2019). Global pathway view analysis of microRNA clusters in myasthenia gravis. Mol. Med. Rep. 19, 2350-2360. doi: 10.3892/mmr.2019.9845

Bogatikov, E., Lindblad, I., Punga, T., and Punga, A. R. (2019). miR-1933-3p is upregulated in skeletal muscles of MuSK+ EAMG mice and affects Impal and Mrp127. Neurosci. Res. 151, 46-52. doi: 10.1016/j.neures.2019.02.003

Bruhova, I., and Auerbach, A. (2017). Molecular recognition at cholinergic synapses: acetylcholine versus choline. J. Physiol. 595, 1253-1261. doi: 10.1113/ JP273291

Buonocore, F., Gerdol, M., Pallavicini, A., Stocchi, V., Randelli, E., Belardinelli, M. C., et al. (2019). Identification, molecular characterization and functional analysis of interleukin (IL)-2 and IL-2like (IL-2L) cytokines in sea bass (Dicentrarchus labrax L.). Cytokine 126:154898. doi: 10.1016/j.cyto.2019. 154898

Cao, Y. L., Dong, W., Li, Y. Z., and Han, W. (2019). MicroRNA-653 inhibits thymocyte proliferation and induces thymocyte apoptosis in have proposed that the silencing of miR-155 could reduce the translocation of nuclear factor (NF)- $\kappa B$ into the nucleus, making it a promising target for the treatment of MG. However, an additional challenge exists for the use of miRNA mimics or antagomir in the treatment of MG due to miRNA degradation. Moreover, although virus vector-mediated, liposome-based, and gold nanoparticle delivery methods may be useful, further studies on miRNA delivery are also needed.

\section{CONCLUSION}

In conclusion, $\mathrm{MG}$ is an autoimmune disease of the nervous system. Its immunological pathogenesis has attracted much attention, particularly the production of autoantibodies, impairment of immune cell function, and the imbalance of cytokines. As important posttranscriptional regulatory factors, miRNAs play a key role in the development of MG. Studying the role of miRNAs in the immunomodulation reaction of MG is of great importance to the clarification of the pathophysiological mechanism underlying this disease. In addition, circulating miRNAs are promising biomarkers for diagnosing and evaluating disease severity, and the development of miRNA-based treatment may also provide a new therapeutic strategy against MG.

\section{AUTHOR CONTRIBUTIONS}

This manuscript was primarily written by LW. The figure was produced by LW and LZ. LZ contributed to the editing of this review. Both authors read and approved the final manuscript.

mice with autoimmune myasthenia gravis by downregulating TRIM9. Neuroimmunomodulation 26, 7-18. doi: 10.1159/000494802

Carthew, R. W., and Sontheimer, E. J. (2009). Origins and mechanisms of miRNAs and siRNAs. Cell 136, 642-655. doi: 10.1016/j.cell.2009.01.035

Cavalcante, P., Bernasconi, P., and Mantegazza, R. (2012). Autoimmune mechanisms in myasthenia gravis. Curr. Opin. Neurol. 25, 621-629. doi: 10. 1097/WCO.0b013e328357a829

Cavalcante, P., Mizrachi, T., Barzago, C., Scandiffio, L., Bortone, F., Bonanno, S., et al. (2019). MicroRNA signature associated with treatment response in myasthenia gravis: a further step towards precision medicine. Pharmacol. Res. 148:104388. doi: 10.1016/j.phrs.2019.104388

Chang, T., Leite, M. I., Senanayake, S., Gunaratne, P. S., Gamage, R., Riffsy, M. T., et al. (2014). Clinical and serological study of myasthenia gravis using both radioimmunoprecipitation and cell-based assays in a South Asian population. J. Neurol. Sci. 343, 82-87. doi: 10.1016/j.jns.2014.05.037

Cheng, Z., Qiu, S., Jiang, L., Zhang, A., Bao, W., Liu, P., et al. (2013). MiR-320a is downregulated in patients with myasthenia gravis and modulates inflammatory cytokines production by targeting mitogen-activated protein kinase 1. J. Clin. Immunol. 33, 567-576. doi: 10.1007/s10875-012-9834-5

Chunjie, N., Huijuan, N., Zhao, Y., Jianzhao, W., and Xiaojian, Z. (2015). Disease-specific signature of serum miR-20b and its targets IL- 8 and IL-25, in myasthenia gravis patients. Eur. Cytokine Netw. 26, 61-66. doi: 10.1684/ecn. 2015.0367

Colamatteo, A., Micillo, T., Bruzzaniti, S., Fusco, C., Garavelli, S., De Rosa, V., et al. (2019). Metabolism and autoimmune responses: the microRNA connection. Front. Immunol. 10:1969. doi: 10.3389/fimmu.2019.01969

Cordiglieri, C., Marolda, R., Franzi, S., Cappelletti, C., Giardina, C., Motta, T., et al. (2014). Innate immunity in myasthenia gravis thymus: pathogenic effects 
of Toll-like receptor 4 signaling on autoimmunity. J. Autoimmun. 52, 74-89. doi: 10.1016/j.jaut.2013.12.013

Cron, M. A., Maillard, S., Delisle, F., Samson, N., Truffault, F., Foti, M., et al. (2018). Analysis of microRNA expression in the thymus of Myasthenia Gravis patients opens new research avenues. Autoimmun. Rev. 17, 588-600. doi: 10. 1016/j.autrev.2018.01.008

Cron, M. A., Maillard, S., Truffault, F., Gualeni, A. V., Gloghini, A., Fadel, E., et al. (2019). Causes and consequences of miR-150-5p dysregulation in myasthenia gravis. Front. Immunol. 10:539. doi: 10.3389/fimmu.2019.00539

Ferrante, M., and Conti, G. O. (2017). Environment and neurodegenerative diseases: an update on miRNA role. Microrna 6, 157-165. doi: 10.2174/ 2211536606666170811151503

Fu, J., Peng, L., Tao, T., Chen, Y., Li, Z., and Li, J. (2019). Regulatory roles of the miR-200 family in neurodegenerative diseases. Biomed. Pharmacother. 119:109409. doi: 10.1016/j.biopha.2019.109409

Gilhus, N. E. (2016). Myasthenia gravis. N. Engl. J. Med. 375, 2570-2581.

Gilhus, N. E., and Verschuuren, J. J. (2015). Myasthenia gravis: subgroup classification and therapeutic strategies. Lancet Neurol. 14, 1023-1036. doi: 10.1016/S1474-4422(15)00145-3

Guptill, J. T., Sanders, D. B., and Evoli, A. (2011). Anti-MuSK antibody myasthenia gravis: clinical findings and response to treatment in two large cohorts. Muscle Nerve 44, 36-40. doi: 10.1002/mus.22006

Ha, M., and Kim, V. N. (2014). Regulation of microRNA biogenesis. Nat. Rev. Mol. Cell Biol. 15, 509-524.

Hajjari, S. N., Mehdizadeh, M., Sadigh-Eteghad, S., Shanehbandi, D., Teimourian, S., and Baradaran, B. (2017). Secretases-related miRNAs in Alzheimer's disease: new approach for biomarker discovery. Neurol. Sci. 38, 1921-1926. doi: 10. 1007/s10072-017-3086-3

Harris, L., Aban, I. B., Xin, H., and Cutter, G. (2019). Employment in refractory myasthenia gravis: a myasthenia gravis foundation of America registry analysis. Muscle Nerve 60, 700-706. doi: 10.1002/mus.26694

Hoch, W., Mcconville, J., Helms, S., Newsom-Davis, J., Melms, A., and Vincent, A. (2001). Auto-antibodies to the receptor tyrosine kinase MuSK in patients with myasthenia gravis without acetylcholine receptor antibodies. Nat. Med. 7, 365-368. doi: $10.1038 / 85520$

Hromadnikova, I., Li, S., Kotlabova, K., and Dickinson, A. M. (2016). Influence of in vitro IL-2 or IL-15 alone or in combination with Hsp 70 derived 14-Mer peptide (TKD) on the expression of NK cell activatory and inhibitory receptors on peripheral blood T cells, B cells and NKT cells. PLoS One 11:e0151535. doi: 10.1371/journal.pone.0151535

Huang, J., Zhang, T., Wang, H., and Zhao, Y. (2018). Treatment of experimental autoimmune myasthenia gravis rats with FTY720 and its effect on Th1/Th2 cells. Mol. Med. Rep. 17, 7409-7414. doi: 10.3892/mmr.2018.8768

Huijbers, M. G., Vergoossen, D. L., Fillie-Grijpma, Y. E., Van Es, I. E., Koning, M. T., Slot, L. M., et al. (2019). MuSK myasthenia gravis monoclonal antibodies: valency dictates pathogenicity. Neurol. Neuroimmunol. Neuroinflamm. 6:e547. doi: 10.1212/NXI.0000000000000547

Jiang, L., Cheng, Z., Qiu, S., Que, Z., Bao, W., Jiang, C., et al. (2012). Altered let7 expression in myasthenia gravis and let-7c mediated regulation of IL-10 by directly targeting IL-10 in Jurkat cells. Int. Immunopharmacol. 14, 217-223. doi: 10.1016/j.intimp.2012.07.003

Jin, F., and Xing, J. (2017). Circulating pro-angiogenic and anti-angiogenic microRNA expressions in patients with acute ischemic stroke and their association with disease severity. Neurol. Sci. 38, 2015-2023. doi: 10.1007/ s10072-017-3071-x

Josefowicz, S. Z., Lu, L. F., and Rudensky, A. Y. (2012). Regulatory T cells: mechanisms of differentiation and function. Annu. Rev. Immunol. 30, 531-564. doi: 10.1146/annurev.immunol.25.022106.141623

Karakatsani, A., Marichal, N., Urban, S., Kalamakis, G., Ghanem, A., Schick, A., et al. (2017). Neuronal LRP4 regulates synapse formation in the developing CNS. Development 144, 4604-4615. doi: 10.1242/dev.15 0110

Khan, U., and Ghazanfar, H. (2018). T lymphocytes and autoimmunity. Int. Rev. Cell Mol. Biol. 341, 125-168.

Kim, T., Mehta, S. L., Morris-Blanco, K. C., Chokkalla, A. K., Chelluboina, B., Lopez, M., et al. (2018). The microRNA miR-7a-5p ameliorates ischemic brain damage by repressing alpha-synuclein. Sci. Signal. 11:eaat4285. doi: 10.1126/ scisignal.aat 4285
Kohler, S., Keil, T. O. P., Hoffmann, S., Swierzy, M., Ismail, M., Ruckert, J. C., et al. (2017). CD4(+) FoxP3(+) T regulatory cell subsets in myasthenia gravis patients. Clin. Immunol. 179, 40-46. doi: 10.1016/j.clim.2017.03.003

Koneczny, I., and Herbst, R. (2019). Myasthenia gravis: pathogenic effects of autoantibodies on neuromuscular architecture. Cells 8:671. doi: 10.3390/ cells 8070671

Kumar, P., Lele, S. S., Ragothaman, V. K., Raghunathan, D., Epstein, A. L., Chiba, S., et al. (2019). OX40L-JAG1-induced expansion of lineage-stable regulatory $\mathrm{T}$ cells involves noncanonical NF-kappaB signaling. J. Immunol. 203, 3225-3236. doi: 10.4049/jimmunol.1900530

Li, J., Qiu, D., Chen, Z., Du, W., Liu, J., and Mo, X. (2016). Altered expression of miR-125a-5p in thymoma-associated myasthenia gravis and its downregulation of foxp3 expression in Jurkat cells. Immunol. Lett. 172, 47-55. doi: 10.1016/j.imlet.2016.02.005

Li, J., Qiu, D., Chen, Z., Du, W., Liu, J., and Mo, X. (2018). miR-548k regulates CXCL13 expression in myasthenia gravis patients with thymic hyperplasia and in Jurkat cells. J. Neuroimmunol. 320, 125-132. doi: 10.1016/j.jneuroim.2018. 03.021

Li, S. C., Shi, H., Khan, M., Caplin, M., Meyer, T., Oberg, K., et al. (2015). Roles of miR-196a on gene regulation of neuroendocrine tumor cells. Mol. Cell. Endocrinol. 412, 131-139. doi: 10.1016/j.mce.2015.06.003

Liu, X., Luo, M., Meng, H., Zeng, Q., Xu, L., Hu, B., et al. (2019). MiR-181a regulates $\mathrm{CD} 4(+) \mathrm{T}$ cell activation and differentiation by targeting IL-2 in the pathogenesis of myasthenia gravis. Eur. J. Immunol. doi: 10.1002/eji.201848007 [Epub ahead of print].

Liu, X. F., Wang, R. Q., Hu, B., Luo, M. C., Zeng, Q. M., Zhou, H., et al. (2016). MiR15 a contributes abnormal immune response in myasthenia gravis by targeting CXCL10. Clin. Immunol. 164, 106-113. doi: 10.1016/j.clim.2015.12.009

Lu, J., Yan, M., Wang, Y., Zhang, J., Yang, H., Tian, F. F., et al. (2013). Altered expression of miR-146a in myasthenia gravis. Neurosci. Lett. 555, 85-90. doi: 10.1016/j.neulet.2013.09.014

Mantegazza, R., and Antozzi, C. (2018). When myasthenia gravis is deemed refractory: clinical signposts and treatment strategies. Ther. Adv. Neurol. Disord. 11:1756285617749134. doi: 10.1177/1756285617749134

Mantegazza, R., Bernasconi, P., and Cavalcante, P. (2018). Myasthenia gravis: from autoantibodies to therapy. Curr. Opin. Neurol. 31, 517-525. doi: 10.1097/WCO. 0000000000000596

Meniailo, M. E., Malashchenko, V. V., Shmarov, V. A., Gazatova, N. D., Melashchenko, O. B., Goncharov, A. G., et al. (2018). Interleukin-8 favors pro-inflammatory activity of human monocytes/macrophages. Int. Immunopharmacol. 56, 217-221. doi: 10.1016/j.intimp.2018.01.036

Meriggioli, M. N., and Sanders, D. B. (2012). Muscle autoantibodies in myasthenia gravis: beyond diagnosis? Expert Rev. Clin. Immunol. 8, 427-438. doi: 10.1586/ eci. 12.34

Michlewski, G., and Caceres, J. F. (2019). Post-transcriptional control of miRNA biogenesis. RNA 25, 1-16. doi: 10.1261/rna.068692.118

Molin, C. J., Sabre, L., Weis, C. A., Punga, T., and Punga, A. R. (2018). Thymectomy lowers the myasthenia gravis biomarker miR-150-5p. Neurol. Neuroimmunol. Neuroinflamm. 5:e450. doi: 10.1212/NXI.0000000000000450

Morren, J., and Li, Y. (2018). Myasthenia gravis with muscle-specific tyrosine kinase antibodies: a narrative review. Muscle Nerve 58, 344-358. doi: 10.1002/ mus.26107

Niendorf, E. R., Parker, J. A., Yechoor, V., Garber, J. R., and Boiselle, P. M. (2005). Thymic hyperplasia in thyroid cancer patients. J. Thorac. Imaging 20, 1-4. doi: 10.1097/01.rti.0000154075.06324.14

Nogales-Gadea, G., Ramos-Fransi, A., Suarez-Calvet, X., Navas, M., Rojas-Garcia, R., Mosquera, J. L., et al. (2014). Analysis of serum miRNA profiles of myasthenia gravis patients. PLoS One 9:e91927. doi: 10.1371/journal.pone. 0091927

Ohnari, K., Okada, K., Higuchi, O., Matsuo, H., and Adachi, H. (2018). Lateonset myasthenia gravis accompanied by amyotrophic lateral sclerosis with antibodies against the acetylcholine receptor and low-density lipoprotein receptor-related protein 4. Intern. Med. 57, 3021-3024. doi: 10.2169/ internalmedicine.0966-18

Park, K. H., Waters, P., Woodhall, M., Lang, B., Smith, T., Sung, J. J., et al. (2018). Myasthenia gravis seronegative for acetylcholine receptor antibodies in South Korea: autoantibody profiles and clinical features. PLoS One 13:e0193723. doi: 10.1371/journal.pone.0193723 
Patel, A. A., Ganepola, G. A. P., Rutledge, J. R., and Chang, D. H. (2019). The potential role of dysregulated miRNAs in Alzheimer's disease pathogenesis and progression. J. Alzheimers Dis. 67, 1123-1145. doi: 10.3233/JAD-181078

Pianta, S., Bonassi Signoroni, P., Muradore, I., Rodrigues, M. F., Rossi, D., Silini, A., et al. (2015). Amniotic membrane mesenchymal cells-derived factors skew $\mathrm{T}$ cell polarization toward Treg and downregulate Th1 and Th17 cells subsets. Stem Cell Rev. Rep. 11, 394-407. doi: 10.1007/s12015-014-9558-4

Pohanka, M. (2019). Inhibitors of cholinesterases in the pharmacology, the current trends. Mini Rev. Med. Chem. doi: 10.2174/1389557519666191018170908 [Epub ahead of print].

Punga, A. R., Andersson, M., Alimohammadi, M., and Punga, T. (2015). Disease specific signature of circulating miR-150-5p and miR-21-5p in myasthenia gravis patients. J. Neurol. Sci. 356, 90-96. doi: 10.1016/j.jns.2015.06.019

Punga, A. R., and Punga, T. (2018). Circulating microRNAs as potential biomarkers in myasthenia gravis patients. Ann. N. Y. Acad. Sci. 1412, 33-40. doi: 10.1111/ nyas. 13510

Punga, T., Bartoccioni, E., Lewandowska, M., Damato, V., Evoli, A., and Punga, A. R. (2016). Disease specific enrichment of circulating let-7 family microRNA in MuSK+ myasthenia gravis. J. Neuroimmunol. 292, 21-26. doi: 10.1016/j. jneuroim.2016.01.003

Quinlan, S., Kenny, A., Medina, M., Engel, T., and Jimenez-Mateos, E. M. (2017). MicroRNAs in neurodegenerative diseases. Int. Rev. Cell Mol. Biol. 334, 309343. doi: 10.1016/bs.ircmb.2017.04.002

Roper, J., Fleming, M. E., Long, B., and Koyfman, A. (2017). Myasthenia gravis and crisis: evaluation and management in the emergency department. J. Emerg. Med. 53, 843-853. doi: 10.1016/j.jemermed.2017.06.009

Sabre, L., Guptill, J. T., Russo, M., Juel, V. C., Massey, J. M., Howard, J. F., et al. (2018a). Circulating microRNA plasma profile in MuSK+ myasthenia gravis. J. Neuroimmunol. 325, 87-91. doi: 10.1016/j.jneuroim.2018.10.003

Sabre, L., Maddison, P., Sadalage, G., Ambrose, P. A., and Punga, A. R. (2018b). Circulating microRNA miR-21-5p, miR-150-5p and miR-30e-5p correlate with clinical status in late onset myasthenia gravis. J. Neuroimmunol. 321, 164-170. doi: 10.1016/j.jneuroim.2018.05.003

Sabre, L., Maddison, P., Wong, S. H., Sadalage, G., Ambrose, P. A., Plant, G. T., et al. (2019). miR-30e-5p as predictor of generalization in ocular myasthenia gravis. Ann. Clin. Transl. Neurol. 6, 243-251. doi: 10.1002/acn3.692

Sengupta, M., Wang, B. D., Lee, N. H., Marx, A., Kusner, L. L., and Kaminski, H. J. (2018). MicroRNA and mRNA expression associated with ectopic germinal centers in thymus of myasthenia gravis. PLoS One 13:e205464. doi: 10.1371/ journal.pone. 0205464

Shen, C., Lu, Y., Zhang, B., Figueiredo, D., Bean, J., Jung, J., et al. (2013). Antibodies against low-density lipoprotein receptor-related protein 4 induce myasthenia gravis. J. Clin. Invest. 123, 5190-5202. doi: 10.1172/JCI66039

Shi, L., Liu, T., Zhang, M., Guo, Y., Song, C., Song, D., et al. (2015). miR$15 \mathrm{~b}$ is downregulated in myasthenia gravis patients and directly regulates the expression of interleukin-15 (IL-15) in experimental myasthenia gravis mice. Med. Sci. Monit. 21, 1774-1780. doi: 10.12659/MSM.893458

Sieb, J. P. (2014). Myasthenia gravis: an update for the clinician. Clin. Exp. Immunol. 175, 408-418. doi: 10.1111/cei.12217

Souto, E. B., Lima, B., Campos, J. R., Martins-Gomes, C., Souto, S. B., and Silva, A. M. (2019). Myasthenia gravis: state of the art and new therapeutic strategies. J. Neuroimmunol. 337:577080. doi: 10.1016/j.jneuroim.2019.577080

Szczudlik, P., Szyluk, B., Lipowska, M., Ryniewicz, B., Kubiszewska, J., Dutkiewicz, M., et al. (2014). Antititin antibody in early- and late-onset myasthenia gravis. Acta Neurol. Scand. 130, 229-233. doi: 10.1111/ane. 12271
Tuzun, E., Allman, W., Ulusoy, C., Yang, H., and Christadoss, P. (2012). Novel animal models of acetylcholine receptor antibody-related myasthenia gravis. Ann. N. Y. Acad. Sci. 1274, 133-139. doi: 10.1111/j.1749-6632.2012.06773.x

Vanhanen, R., Tuulasvaara, A., Mattila, J., Patila, T., and Arstila, T. P. (2018). Common gamma chain cytokines promote regulatory $\mathrm{T}$ cell development and survival at the $\mathrm{CD} 4(+) \mathrm{CD} 8(+)$ stage in the human thymus. Scand. J. Immunol. 88:e12681. doi: $10.1111 /$ sji.12681

Vincent, A., Palace, J., and Hilton-Jones, D. (2001). Myasthenia gravis. Lancet 357, 2122-2128.

Wang, J., Muheremu, A., Zhang, M., Gong, K., Huang, C., Ji, Y., et al. (2016). MicroRNA-338 and microRNA-21 co-transfection for the treatment of rat sciatic nerve injury. Neurol. Sci. 37, 883-890. doi: 10.1007/s10072-016-2500-6

Wang, J., Zheng, S., Xin, N., Dou, C., Fu, L., Zhang, X., et al. (2013). Identification of novel MicroRNA signatures linked to experimental autoimmune myasthenia gravis pathogenesis: down-regulated miR-145 promotes pathogenetic Th17 cell response. J. Neuroimmune Pharmacol. 8, 1287-1302. doi: 10.1007/s11481-0139498-9

Wang, Y. Z., Tian, F. F., Yan, M., Zhang, J. M., Liu, Q., Lu, J. Y., et al. (2014). Delivery of an miR155 inhibitor by anti-CD20 single-chain antibody into B cells reduces the acetylcholine receptor-specific autoantibodies and ameliorates experimental autoimmune myasthenia gravis. Clin. Exp. Immunol. 176, 207221. doi: $10.1111 /$ cei. 12265

Xie, S., Li, X., Qian, L., Cai, C., Xiao, G., Jiang, S., et al. (2019). An integrated analysis of mRNA and miRNA in skeletal muscle from myostatin-edited Meishan pigs. Genome 62, 305-315. doi: 10.1139/gen-2018-0110

Yeh, J. H., Wang, S. H., Chien, P. J., Shih, C. M., and Chiu, H. C. (2009). Changes in serum cytokine levels during plasmapheresis in patients with myasthenia gravis. Eur. J. Neurol. 16, 1318-1322. doi: 10.1111/j.1468-1331.2009.02729.x

Yin, W., Ouyang, S., Luo, Z., Zeng, Q., Hu, B., Xu, L., et al. (2017). Immature exosomes derived from MicroRNA-146a overexpressing dendritic cells act as antigen-specific therapy for myasthenia gravis. Inflammation 40, 1460-1473. doi: 10.1007/s10753-017-0589-2

Zhang, Y., Guo, M., Xin, N., Shao, Z., Zhang, X., Zhang, Y., et al. (2016). Decreased microRNA miR-181c expression in peripheral blood mononuclear cells correlates with elevated serum levels of IL-7 and IL-17 in patients with myasthenia gravis. Clin. Exp. Med. 16, 413-421. doi: 10.1007/s10238-0150358-1

Zhao, Y. Y., Wang, W. A., and Hu, H. (2015). Treatment with recombinant tissue plasminogen activator alters the microRNA expression profiles in mouse brain after acute ischemic stroke. Neurol. Sci. 36, 1463-1470. doi: 10.1007/s10072015-2149-6

Zuckerman, N. S., Howard, W. A., Bismuth, J., Gibson, K., Edelman, H., BerrihAknin, S., et al. (2010). Ectopic GC in the thymus of myasthenia gravis patients show characteristics of normal GC. Eur. J. Immunol. 40, 1150-1161. doi: 10. 1002/eji.200939914

Conflict of Interest: The authors declare that the research was conducted in the absence of any commercial or financial relationships that could be construed as a potential conflict of interest.

Copyright (c) 2020 Wang and Zhang. This is an open-access article distributed under the terms of the Creative Commons Attribution License (CC BY). The use, distribution or reproduction in other forums is permitted, provided the original author(s) and the copyright owner(s) are credited and that the original publication in this journal is cited, in accordance with accepted academic practice. No use, distribution or reproduction is permitted which does not comply with these terms. 